\title{
Anticipatory Governance in the Technology Sector: Processes, Critiques and Principles for Addressing Grand Challenges in Computing
}

KELLY WIDDICKS, School of Computing and Communications, Lancaster University, UK

BRAN KNOWLES, School of Computing and Communications, Lancaster University, UK

GORDON BLAIR, School of Computing and Communications, Lancaster University, UK

CAROLYN TEN HOLTER, Department of Computer Science, University of Oxford, UK

MARINA JIROTKA, Department of Computer Science, University of Oxford, UK

FEDERICA LUCIVERO, Nuffield Department of Population Health, University of Oxford, UK

GABRIELLE SAMUEL, University of Southampton, UK

HELENA WEBB, Department of Computer Science, University of Oxford, UK

With growing understanding of negative social and environmental impacts of computing technologies and increasingly urgent calls to mitigate these impacts, the sector now faces thorny questions around whether and how to govern computing technologies. This workshop aims to bring together researchers and practitioners across a wide range of disciplines to explore critical perspectives on and solutions to anticipatory governance in the computing sector. We will draw on participants' diverse expertise to develop a practical and ethical governance roadmap that attends to the computing sector's responsibility to mitigate its own contribution to the climate emergency. Having developed strategies within this specific context, we will then produce a set of governance principles that could be useful to mitigate other harms resulting from computing, nominally those pertaining to efforts around responsible AI, data protection, and mis/disinformation.

CCS Concepts: • Social and professional topics; • Human-centered computing $\rightarrow$ Human computer interaction (HCI);

Additional Key Words and Phrases: HCI, technology governance, ethical governance, anticipatory governance, responsible research, responsible innovation

\section{ACM Reference Format:}

Kelly Widdicks, Bran Knowles, Gordon Blair, Carolyn Ten Holter, Marina Jirotka, Federica Lucivero, Gabrielle Samuel, and Helena Webb. 2021. Anticipatory Governance in the Technology Sector: Processes, Critiques and Principles for Addressing Grand Challenges in Computing. In CHI Conference on Human Factors in Computing Systems Extended Abstracts (CHI '21 Extended Abstracts), May 8-13, 2021, Yokohama, Japan. ACM, New York, NY, USA, 8 pages. https://doi .org/10.1145/3411763.3441314

\section{BACKGROUND}

Computing innovations have profound impacts on our society and environment-both good and bad, though often a mix of the two. To ensure ethical design and development processes, the Human-Computer Interaction (HCI) community has been, and continues to, drive agendas such as responsible innovation [2,12], reflective and critical computing [18], and sustainable [4], social justice-orientated [8] and value sensitive design [9] that foreground and critique technology's

Permission to make digital or hard copies of part or all of this work for personal or classroom use is granted without fee provided that copies are not made or distributed for profit or commercial advantage and that copies bear this notice and the full citation on the first page. Copyrights for third-party components of this work must be honored. For all other uses, contact the owner/author(s).

(c) 2021 Copyright held by the owner/author(s).

Manuscript submitted to ACM 
potential positive and negative impacts. Such work is gaining attention in the HCI community, with CHI'21 introducing a new subcommittee for 'Critical and Sustainable' Computing-showing commitment to HCI research around, for example, diversity and inclusion, sustainability, and social justice. Clearly many professionals in the technology sector care about, and are individually attempting to alleviate, technology's negative consequences; but certain harms require a coordinated effort and sector-wide commitment to be adequately mitigated.

Laws, policies and regulations help protect society from wrongdoings, and the HCI community has explored the link between policy and HCI and how they can inform each other [14, 15, 21, 24]; most recently, CHI workshops in this domain have looked to explore 'Soft Law' mechanisms for privacy regulations [23] and HCI's role in criminal justice [3]. Less explored, however, are the mechanisms that might need to be developed within the computing sector itself to ensure responsible innovation [28]. How might we, as a community, govern computing? By governance in this context, we refer to 'anticipatory governance' i.e. the management of emerging technologies, encouraging stakeholder reflection of their roles in such technologies [11] and determining what organisations or procedures are needed to ensure accountability and responsibility in computing research and development [27].

Such governance implies a constraint on innovation and this notion is generally unpopular in computing, yet constraint is exactly what is required in some cases where computing can have significant negative consequencesparticularly through major challenges the sector faces (see [13]). There is perhaps no clearer case of this need for governance than the issue of climate emergency and computing's own growing carbon footprint. To better explore practical strategies for anticipatory governance, and to produce tangible impact in a space in need of urgent solutions, we will focus this workshop around the climate emergency-though with a view to formulating more broadly transferable insights into other grand challenges in computing through a set of principles for anticipatory governance in the sector.

Relevant context with respect to the challenge of mitigating climate impacts is as follows. Information and Communication Technologies (ICT) have an environmental impact amounting to 4-5\% of our global $\mathrm{CO}_{2}$ emissions [1], and this cannot be simply ignored due to the various benefits computing affords. Historically, technology innovation has risen alongside global emissions, and with growth trends such as Artificial Intelligence (AI), the Internet of Things, and Blockchain, it is likely this trend will continue unless there is broad political and industrial action. For a sustainable environment, the Paris Agreement sets out unambiguous targets to reduce carbon by $42 \%$ by $2030,72 \%$ by 2040 , and by $91 \%$ by 2050 to keep global warming under 1.5 degrees Celsius-a critical tipping point for the habitability of the planet. Like all other sectors in the global economy, the technology sector should reduce its emissions to meet Paris targets.

But even defining sensible targets for the sector is fraught with difficulty. For one, the European climate strategy relies heavily on technology-enabled efficiency savings across the economy. There are reasons to believe that these efficiencies are unlikely to yield carbon savings anywhere near the $15 \%$ as estimated by the European Commission [6], but critically there is no transparent and thorough accounting of the scale of emissions reductions that can be expected from technology. This is key information in deciding whether computing's own footprint needs to grow to deliver economy-wide savings, or at what pace it will need to reduce. Assuming we can arrive at clear targets for the sector, however, how are we going to meet these targets? There are notable examples of climate leadership in the sector, but in the absence of mechanisms to reward this leadership and/or punish companies neglecting their climate responsibilities, there is a de facto competitive advantage for companies who do not meet reductions targets. This indicates a need for anticipatory governance: oversight and enforcement mechanisms that keep technology on track to meet carbon targets.

There is an additional wrinkle, however. There is no reason to assume that all types of computing should reduce carbon footprints by the same margin. For example, there are great number of promising applications of technology toward mitigating the climate crisis-many in service of enabling efficiency (and, theoretically, emissions reductions). Manuscript submitted to ACM 
These efforts include, e.g. using technology to raise awareness about energy consumption, to better understand the changing natural environment, and to track and manage supply chains (see cf. [7, 19]). It would make sense that these innovations receive a greater portion of the carbon budget than technologies that do not contribute toward sustainability. But technologies can be worthy in other ways, and it would be unwise to be too single-minded about what computing deserves prioritisation. This raises a number of difficult questions that must be addressed head-on, for example:

- How can we minimise technology's environmental impacts within the context of a digital economy that thrives through innovation?

- If decisions need to be made about how to spend a limited carbon budget shared across the computing sector, how do we as a global computing community decide which innovations should be disallowed, on what basis, and using what deliberative processes to ensure these decisions are reasonable, well-grounded and equitable?

- What are the implications of disallowing some kinds of technologies, what would this look like in practice, and how could it possibly be enforced?

Through this workshop, we aim to bring together researchers and practitioners across a wide range of disciplines to begin to unpack and explore our community's role in anticipatory governance for computing. Specifically, we will look to create a set of principles for technology governance, exploring questions such as: what processes and procedures the sector should take to govern technology; what different values, stakeholders, lenses and perspectives do we need to consider for ethical governance; what prior case studies of governance or non-governance can we learn from, within and beyond the technology sector; and what role might HCI play in enabling governance? Drawing on socio-technical and responsible research and innovation (cf. $[10,22]$ ), the current landscape of governance across countries and jurisdictions (e.g. data governance in the UK [20], or globally for specific technologies [5]), and project-level challenges of balancing research, innovation and stakeholder participation [16], workshop discussions will elicit a set of governance principles for computing to be able to define and meet sector-wide carbon targets. The extent to which these principles are broadly transferable to the mitigation of other harms resulting from computing will be a topic for reflection.

\section{ORGANISERS}

The organisers of the workshop have diverse yet complementary skill sets and expertise, working within a range of disciplines including computer science, sociology, philosophy and ethics, and bringing together an understanding of socio-technical research and responsible innovation that anticipatory governance in the technology sector requires. Some organisers have previously worked together on publications, projects and workshops, and all organisers have recently collaborated on a grant submission in which computing governance will play a significant part. The workshop outcomes therefore have the potential to form part of a wider project and its impact.

Kelly Widdicks, the main contact for the workshop, is an EPSRC Doctoral Prize researcher at Lancaster University, interested in understanding and mitigating the negative impacts of technology. Her research to date has concerned the sustainability and digital wellbeing implications of HCI design [25, 26], both of which would benefit from technology governance. She is currently working with Knowles and Blair to study how researchers and technologists prioritise and negotiate innovation-providing insights into how technology may be governed in the sector for a sustainable future.

Bran Knowles is a Senior Lecturer in the Data Science Institute at Lancaster University. Her research explores socially responsible and trusted computing across a wide range of contexts. She currently serves on the ACM Europe Technology Policy Committee where she leads the Sustainability standing group and recently authored ACM Europe's official response to the EU Commission's Green Deal (bit.ly/2B2v92e).

Manuscript submitted to ACM 
Gordon Blair is a Distinguished Professor at Lancaster University, and is Co-Director of the recently created Center of Excellence in Environmental Data Science, a joint initiative with UKCEH. He also holds a prestigious EPSRC Senior Fellowship in Digital Technology and Living with Environmental Change (DT/LWEC). His research interests are concerned with how digital innovation can support understanding and management of the natural environment.

Carolyn Ten Holter is a researcher in the Human Centred Computing Group at the University of Oxford's Department of Computer Science. She is part of the Responsible Technology Institute, and her work focuses on the application of responsible innovation (RI) methodologies - including sustainability - to both academic and industrial innovation.

Marina Jirotka is Professor of Human Centred Computing in the Department of Computer Science at the University of Oxford and has been at the forefront of recent work in Responsible Innovation (RI) in the UK and the European Union. She leads an interdisciplinary research group investigating the responsible development of technologies that are more responsive to societal acceptability and desirability. She has recently been awarded an EPSRC Established Career Fellowship on Developing Responsible Robotics for the Digital Economy, and is Director of the Responsible Technology Institute at Oxford.

Federica Lucivero is a Senior Researcher at the Nuffield Department of Population Health. Her expertise spans ethics, social studies of science and technology, and governance of innovation. She has recently published on the moral and ethical aspects related to the environmental impacts of data-driven technologies (cf. [17]).

Gabrielle Samuel is a Senior Research Fellow at the University of Southampton. Her research draws on qualitative methods to explore the ethical, social and regulatory issues associated with new and emerging data-driven health and forensic technologies and methodologies. She has particular interest in AI, genomics, and digital health technologies more generally.

Helena Webb is a Senior Researcher in the Department of Computer Science at the University of Oxford. She investigates the sociology of technology, responsible innovation and Human Computer Interaction. Helena has expertise in exploring the lived experience of technology and identifying mechanisms to ensure innovations better meet societal and environmental needs.

\section{WEBSITE}

Details about the workshop will be available on the following website URL: http://wp. lancs.ac.uk/technology-gove rnance-workshop/. Accepted position papers from participants and outputs of the workshop will be published on this website; this will act as a hub for participants to engage in the workshop materials before and after CHI'2021.

\section{PRE-WORKSHOP PLANS}

Those wishing to attend the workshop will be asked to write a 2-4 page Q\&A paper using our template on the workshop website, answering the following (though with more or less emphasis on particular questions as they see fit):

- What is your background/expertise, and how did you become interested in governance?

- What themes, challenges and/or case studies have you explored in your work?

- What disciplines, concepts or critical lenses (e.g. feminist, post-colonial) might we draw on to ensure that governance is appropriate or ethical?

- What do you think are some concrete strategies for governing the computing sector, and what does success look like?

Manuscript submitted to ACM 
- What do you think are the major challenges associated with governing the environmental impact of computing technologies?

- What do you think participants at this workshop need to know (that you think they might not know) as we work together to develop principles for anticipatory governance in the sector?

- What are you hoping to get out of attending this workshop?

- Is there anything else you would like to tell us?

Once submitted, the organisers will evaluate the position papers and select 15-20 participants that will provide a set of diverse expertise and perspectives for the workshop. Position papers will be made available on the workshop website and participants will be encouraged to familiarise themselves with each others' papers prior to the workshop. The organisers will also use these position papers to make panels on themes emerging from the submissions. Panelists will prepare responses to questions that the organisers have developed based on particularly interesting answers from the Q\&A submissions, and answer questions from the other participants.

\section{WORKSHOP SCHEDULE}

The schedule for the workshop is outlined below.

09:00-09:10 Welcome from the organisers. Workshop organisers will introduce themselves and the schedule.

09:10-09:50 Participant panels. Two simultaneous sessions in breakout rooms, each with two 20-minute panels consisting of 3-5 participants as panelists. Participants will be grouped based on differing viewpoints from overlapping themes discussed in their position papers. The organisers will facilitate the panel discussions, asking participants to expand on their position papers and discuss those of others.

09.50-10:10 Themes. As a whole group, workshop participants will participate in an exercise to identify themes of technology governance developed from the panels. Participants will choose a theme to discuss as a smaller group in the case study roadmap development session, based on their interests and expertise.

10:10-10:30 Scheduled break.

10:30-10:45 Case study: computing and climate change. The roadmap development session will be oriented around how the computing community might define and meet sector-wide carbon targets. Because participants are not expected to be climate change experts, the organisers will introduce the task by delivering a short presentation that provides key facts regarding the computing's current and projected carbon impacts, and specific challenges in devising a carbon strategy for the sector. The presentation will include slides and captioning.

10:45-12:00 Roadmap development. In breakout rooms focusing on the climate change case study and their chosen theme from the ideation session, participants will explore and develop concrete elements that would comprise an anticipatory governance strategy for sustainable computing. Organisers will facilitate note taking and participants will identify a 'group lead' to summarise the discussion back to the whole workshop in the roadmap feedback session.

12:00-13:00 Lunch break.

13:00-13:20 Roadmap feedback. Each of the group leads will feedback to the whole workshop group, providing a short summary of their discussions from the roadmap development session. Participants will be able to ask questions and comment on the groups' ideas.

13:20-14:20 Identifying technology governance principles. A set of questions will be drawn from the prior session which newly constituted discussion groups will then explore in search of broad governance principles. Groups will work independently in breakout rooms, but each capturing their discussions in the same shared Google doc. This 
Google doc will form the basis for a publication output resulting from the workshop.

14:20-14:30 Summary from the organisers and next steps. The organisers will summarise the day and the next steps they will take post-workshop to disseminate the workshop results and technology governance principles. Participants will be encouraged to write-up and disseminate the outcomes from their small group discussions in the roadmap development session, as well as contribute to a publication summarising key governance challenges and principles for computing.

\section{DISTANCE ENGAGEMENT}

We will use Zoom conferencing, with breakout rooms for the participant panels, roadmap development session and governance principles session. Workshop organisers will facilitate note taking in each of the exploration sessions, sharing their notes screen with the rest of the group. Google Docs will be used for online collaborative writing.

\section{POST-WORKSHOP PLANS}

After the workshop, the organisers plan to write-up the set of principles for anticipatory governance in the technology sector as an article for the Human-Computer Interaction community. The article venue would be chosen to be commensurate with the contribution (both size and style) derived from discussions, and taking into account participants' preferences. Possible venues include: ACM Interactions, Communications of the ACM, ACM CHI Conference on Human Factors in Computing Systems, or ACM Transactions on Human-Computer Interaction (ToCHI). All workshop participants will be acknowledged in the publication and they will be sent the article for their information with the option to provide feedback. Participants will also be encouraged to disseminate the outcomes of their roadmap development exercise, e.g. through writing their own discussion papers in their small groups; if participants do this, they will be asked to share their work with the rest of the workshop participants for information or for comment. A mailing list will be created for those interested in technology governance, which the participants can use to share their work. Links to all outputs of the workshop will be added to the workshop website. The workshop outputs may also form part of a wider project to be conducted by the organisers, maximising the impact of the work.

\section{CALL FOR PARTICIPANTS}

This workshop will unpack how we may address a major challenge in the technology sector: how technology should be governed. Drawing on ethical and responsible innovation and focusing on computing and climate change as a case study, this workshop will identify a set of principles required for anticipatory governance in technology.

This virtual workshop will comprise a set of participant panels, where participants can expand on their diverse expertise on problems and opportunities for technology governance; small group work to identify key themes, strategies and principles; and full group discussion and debate.

Those wishing to attend should submit a 2-4 page Q\&A paper regarding technology governance using the Q\&A template on the workshop website: http://wp.lancs.ac.uk/technology-governance-workshop/. Anyone with relevant expertise, within and beyond the technology sector, is welcome to apply. Expertise in climate change or sustainability is not required.

Responses should be submitted to Kelly Widdicks (k.v.widdicks@lancaster.ac.uk) by 1st February 2021 with the subject line "CHI Governance Workshop Submission". Submissions will be reviewed and selected to form an interesting variety of perspectives for the workshop. Notifications of submission outcomes will be sent by 21st February 2021 Accepted participants are expected to register and attend the workshop alongside at least one day of the conference. Manuscript submitted to ACM 
Organisers: Kelly Widdicks, Bran Knowles, Gordon Blair, Carolyn Ten Holter, Marina Jirotka, Federica Lucivero, Gabrielle Samuel, and Helena Webb.

\section{ACKNOWLEDGMENTS}

This work is partially supported by: Kelly Widdicks' Engineering and Physical Sciences Research Council (EPSRC) Doctoral Prize (grant no. EP/R513076/1); Gordon Blair's EPSRC Senior Fellowship in Digital Technology and Living with Environmental Change (grant no. EP/P002285/1); the EPSRC 'RoboTIPS' study on responsible robotics (EP/S005099/1), funding Helena Webb; and the Wellcome Centre for Ethics and Humanities (grant no. 203132), funding Federica Lucivero.

\section{REFERENCES}

[1] Anders SG Andrae. 2020. Hypotheses for primary energy use, electricity use and CO2 emissions of global computing and its shares of the total between 2020 and 2030. WSEAS Transactions on Power Systems (2020).

[2] Oliver Bates, Kathy New, Samantha Mitchell-Finnigan, Matthew Louis Mauriello, Christian Remy, Roy Bendor, Samuel Mann, Simran Chopra, Adrian K. Clear, and Chris Preist. 2019. Towards a Responsible Innovation Agenda for HCI. In Extended Abstracts of the 2019 CHI Conference on Human Factors in Computing Systems (Glasgow, Scotland Uk) (CHI EA '19). Association for Computing Machinery, New York, NY, USA, 1-8. https://doi.org/10.1145/3290607.3299017

[3] Rosanna Bellini, Nicola Dell, Monica Whitty, Debasis Bhattacharya, David Wall, and Pamela Briggs. 2020. Crime and/or Punishment: Joining the Dots between Crime, Legality and HCI. In Extended Abstracts of the 2020 CHI Conference on Human Factors in Computing Systems (Honolulu, HI, USA) (CHI EA '20). Association for Computing Machinery, New York, NY, USA, 1-8. https://doi .org/10.1145/3334480.3375176

[4] Eli Blevis. 2007. Sustainable Interaction Design: Invention \& Disposal, Renewal \& Reuse. In Proceedings of the SIGCHI Conference on Human Factors in Computing Systems (San Jose, California, USA) (CHI '07). Association for Computing Machinery, New York, NY, USA, 503-512. https: //doi.org/10.1145/1240624.1240705

[5] James Butcher and Irakli Beridze. 2019. What is the State of Artificial Intelligence Governance Globally? The RUSI Fournal 164, 5-6 (2019), 88-96. https://doi.org/10.1080/03071847.2019.1694260 arXiv:https://doi.org/10.1080/03071847.2019.1694260

[6] European Commission. 2020. Supporting the green transition. https://ec.europa.eu/commission/presscorner/detail/en/fs_20_ 281-accessedMarch2020

[7] Carl DiSalvo, Phoebe Sengers, and Hrönn Brynjarsdóttir. 2010. Mapping the Landscape of Sustainable HCI. In Proceedings of the SIGCHI Conference on Human Factors in Computing Systems (Atlanta, Georgia, USA) (CHI '10). Association for Computing Machinery, New York, NY, USA, $1975-1984$. https://doi.org/10.1145/1753326.1753625

[8] Lynn Dombrowski, Ellie Harmon, and Sarah Fox. 2016. Social Justice-Oriented Interaction Design: Outlining Key Design Strategies and Commitments. In Proceedings of the 2016 ACM Conference on Designing Interactive Systems (Brisbane, QLD, Australia) (DIS '16). Association for Computing Machinery, New York, NY, USA, 656-671. https://doi.org/10.1145/2901790.2901861

[9] Batya Friedman, Peter H Kahn, and Alan Borning. 2008. Value sensitive design and information systems. The handbook of information and computer ethics (2008), 69-101.

[10] Barbara Grimpe, Mark Hartswood, and Marina Jirotka. 2014. Towards a Closer Dialogue between Policy and Practice: Responsible Design in HCI. In Proceedings of the SIGCHI Conference on Human Factors in Computing Systems (Toronto, Ontario, Canada) (CHI '14). Association for Computing Machinery, New York, NY, USA, 2965-2974. https://doi.org/10.1145/2556288.2557364

[11] David H Guston. 2014. Understanding 'anticipatory governance'. Social Studies of Science 44, 2 (2014), 218-242. https: //doi.org/10.1177/ 0306312713508669 arXiv:https://doi.org/10.1177/0306312713508669 PMID: 24941612.

[12] Marina Jirotka, Barbara Grimpe, Bernd Stahl, Grace Eden, and Mark Hartswood. 2017. Responsible Research and Innovation in the Digital Age. Commun. ACM 60, 5 (April 2017), 62-68. https://doi.org/10.1145/3064940

[13] Lorraine Kisselburgh, Michel Beaudouin-Lafon, Lorrie Cranor, Jonathan Lazar, and Vicki L. Hanson. 2020. HCI Ethics, Privacy, Accessibility, and the Environment: A Town Hall Forum on Global Policy Issues. In Extended Abstracts of the 2020 CHI Conference on Human Factors in Computing Systems (Honolulu, HI, USA) (CHI EA '20). Association for Computing Machinery, New York, NY, USA, 1-6. https://doi.org/10.1145/3334480. 3381067

[14] Jonathan Lazar, Julio Abascal, Simone Barbosa, Jeremy Barksdale, Batya Friedman, Jens Grossklags, Jan Gulliksen, Jeff Johnson, Tom McEwan, Loïc Martínez-Normand, Wibke Michalk, Janice Tsai, Gerrit van der Veer, Hans von Axelson, Ake Walldius, Gill Whitney, Marco Winckler, Volker Wulf, Elizabeth F. Churchill, Lorrie Cranor, Janet Davis, Alan Hedge, Harry Hochheiser, Juan Pablo Hourcade, Clayton Lewis, Lisa Nathan, Fabio Paterno, Blake Reid, Whitney Quesenbery, Ted Selker, and Brian Wentz. 2016. Human-Computer Interaction and International Public Policymaking: A Framework for Understanding and Taking Future Actions. Foundations and Trends® in Human-Computer Interaction 9, 2 (2016), 69-149. https://doi.org/10.1561/1100000062 
[15] Jonathan Lazar, Simone Barbosa, Jan Gulliksen, Tom McEwan, Loic Martinez Normand, Philippe Palanque, Raquel Prates, Janice Tsai, Marco Winckler, and Volker Wulf. 2013. Workshop on Engaging the Human-Computer Interaction Community with Public Policymaking Internationally. In CHI '13 Extended Abstracts on Human Factors in Computing Systems (Paris, France) (CHI EA '13). Association for Computing Machinery, New York, NY, USA, 3279-3282. https://doi .org/10.1145/2468356.2479666

[16] Susan Lechelt, Chris Elsden, Ingi Helgason, Inge Panneels, Michael Smyth, Chris Speed, and Melissa Terras. 2019. How Can We Balance Research, Participation and Innovation as HCI Researchers?. In Proceedings of the Halfway to the Future Symposium 2019 (Nottingham, United Kingdom) (HTTF 2019). Association for Computing Machinery, New York, NY, USA, Article 10, 4 pages. https://doi.org/10.1145/3363384.3363394

[17] Federica Lucivero, Gabrielle Samuel, Gordon Blair, Sarah J Darby, Tina Fawcett, Mike Hazas, Carolyn Ten Holter, Marina Jirotka, Michael Parker Helena Webb, and Hang Yuan. 2020. Data-driven unsustainability? An interdisciplinary perspective on governing the environmental impacts of a data-driven society. (2020). https://dx.doi.org/10.2139/ssrn. 3631331

[18] Phoebe Sengers, Kirsten Boehner, Shay David, and Joseph 'Jofish' Kaye. 2005. Reflective Design. In Proceedings of the 4th Decennial Conference on Critical Computing: Between Sense and Sensibility (Aarhus, Denmark) (CC '05). Association for Computing Machinery, New York, NY, USA, 49-58. https://doi.org/10.1145/1094562.1094569

[19] M. Six Silberman, Lisa Nathan, Bran Knowles, Roy Bendor, Adrian Clear, Maria Håkansson, Tawanna Dillahunt, and Jennifer Mankoff. 2014. Next Steps for Sustainable HCI. Interactions 21, 5 (Sept. 2014), 66-69. https://doi.org/10.1145/2651820

[20] The Royal Society. 2020. The UK data governance landscape. Technical Report. The Royal Society. https://royalsociety.org/-/media/p olicy/projects/data-governance/uk-data-governance-explainer.pdf, accessed October 2020.

[21] Anne Spaa, Abigail Durrant, Chris Elsden, and John Vines. 2019. Understanding the Boundaries between Policymaking and HCI. In Proceedings of the 2019 CHI Conference on Human Factors in Computing Systems (Glasgow, Scotland Uk) (CHI '19). Association for Computing Machinery, New York, NY, USA, 1-15. https://doi.org/10.1145/3290605.3300314

[22] Jack Stilgoe, Richard Owen, and Phil Macnaghten. 2013. Developing a framework for responsible innovation. Research Policy 42, 9 (2013), 1568 1580. https://doi.org/10.1016/j.respol.2013.05.008

[23] Eva Thelisson, Kshitij Sharma, Hanan Salam, and Virginia Dignum. 2018. The General Data Protection Regulation: An Opportunity for the HCI Community?. In Extended Abstracts of the 2018 CHI Conference on Human Factors in Computing Systems (Montreal QC, Canada) (CHI EA '18). Association for Computing Machinery, New York, NY, USA, 1-8. https://doi .org/10.1145/3170427.3170632

[24] Vanessa Thomas, Christian Remy, Mike Hazas, and Oliver Bates. 2017. HCI and Environmental Public Policy: Opportunities for Engagement. In Proceedings of the 2017 CHI Conference on Human Factors in Computing Systems (Denver, Colorado, USA) (CHI '17). Association for Computing Machinery, New York, NY, USA, 6986-6992. https://doi.org/10.1145/3025453.3025579

[25] Kelly Widdicks. 2020. When the Good Turns Ugly: Speculating Next Steps for Digital Wellbeing Tools. In Proceedings of the 2020 Nordic Conference on Human-Computer Interaction. Association for Computing Machinery.

[26] Kelly Widdicks, Mike Hazas, Oliver Bates, and Adrian Friday. 2019. Streaming, Multi-Screens and YouTube: The New (Unsustainable) Ways of Watching in the Home. In Proceedings of the 2019 CHI Conference on Human Factors in Computing Systems (Glasgow, Scotland Uk) (CHI '19). Association for Computing Machinery, New York, NY, USA, 1-13. https://doi .org/10.1145/3290605.3300696

[27] Alan F. T. Winfield and Marina Jirotka. 2017. The Case for an Ethical Black Box. In Towards Autonomous Robotic Systems, Yang Gao, Saber Fallah, Yaochu Jin, and Constantina Lekakou (Eds.). Springer International Publishing, Cham, 262-273.

[28] Alan F. T. Winfield and Marina Jirotka. 2018. Ethical governance is essential to building trust in robotics and artificial intelligence systems. Philosophical Transactions of the Royal Society A: Mathematical, Physical and Engineering Sciences 376, 2133 (2018), 20180085. https://doi .org/ 10.1098/rsta.2018.0085 arXiv:https://royalsocietypublishing.org/doi/pdf/10.1098/rsta.2018.0085 